\title{
Suppression of STAT5A increases chemotherapeutic sensitivity in imatinib-resistant and imatinib-sensitive K562 cells
}

\author{
Buket Kosova, Burcin Tezcanli, Huseyin Atakan Ekiz, Zeynep Cakir, Nur Selvi, \\ Aysegul Dalmizrak, Melis Kartal, Ufuk Gunduz \& Yusuf Baran
}

To cite this article: Buket Kosova, Burcin Tezcanli, Huseyin Atakan Ekiz, Zeynep Cakir, Nur Selvi, Aysegul Dalmizrak, Melis Kartal, Ufuk Gunduz \& Yusuf Baran (2010) Suppression of STAT5A increases chemotherapeutic sensitivity in imatinib-resistant and imatinib-sensitive K562 cells, Leukemia \& Lymphoma, 51:10, 1895-1901, DOI: 10.3109/10428194.2010.507830

To link to this article: http://dx.doi.org/10.3109/10428194.2010.507830

曲 Published online: 20 Sep 2010.

Submit your article to this journal $₫$

Џ Article views: 104

Q View related articles $\sqsubset$ 


\title{
Suppression of STAT5A increases chemotherapeutic sensitivity in imatinib-resistant and imatinib-sensitive $\mathrm{K} 562$ cells
}

\author{
BUKET KOSOVA ${ }^{1}$, BURCIN TEZCANLI ${ }^{1}$, HUSEYIN ATAKAN EKIZ ${ }^{2}$, ZEYNEP CAKIR ${ }^{2}$, \\ NUR SELVI ${ }^{1}$, AYSEGUL DALMIZRAK ${ }^{1}$, MELIS KARTAL $^{2}$, UFUK GUNDUZ $^{3}$, \& \\ YUSUF BARAN ${ }^{2}$ \\ ${ }^{1}$ Department of Medical Biology, Medical School, Ege University, Izmir, Turkey, ${ }^{2}$ Department of Molecular Biology and \\ Genetics, Izmir Institute of Technology, Izmir, Turkey, and ${ }^{3}$ Department of Molecular Biology and Genetics, Middle East \\ Technical University, Ankara, Turkey
}

(Received 18 May 2010; revised 15 fune 2010; accepted 6 fuly 2010)

\begin{abstract}
STAT proteins are cytoplasmic transcription factors that are involved in the regulation of numerous cellular activities such as cell growth, differentiation, and survival. In this study, we aimed to identify the expression pattern of STAT genes in imatinib-sensitive and -resistant K562 cells, and further, to reveal the effects of STAT5A siRNA knockdown on cell growth and apoptosis induction. The XTT cell proliferation assay showed that both sensitive and resistant K562 cells were sensitized to imatinib upon transfection with STAT5A siRNA. Caspase-3 enzyme activity was increased significantly in both cells. These results may open up new opportunities to overcome chemotherapeutic resistance in leukemia.
\end{abstract}

Keywords: Chronic myeloid leukemia (CML), chemotherapeutic resistance, reversal of resistance, imatinib, STAT5A, siRNA knockdown

\section{Introduction}

STATs (signal transducers and activator of transcription proteins) are latent cytoplasmic transcription factors that, upon activation, carry information coming from outside the cell into the nucleus. Extensive studies in mammalian systems have revealed that STATs consist of seven structural and functional homolog family members: STAT1, STAT2, STAT3, STAT4, STAT5A, STAT5B, and STAT6 [1,2]. STAT activation is normally achieved by phosphorylation of specific tyrosine residues, accomplished either by ligand-activated receptor tyrosine kinases such as the epidermal growth factor receptor (EGFR) or by receptors lacking intrinsic tyrosine kinase ability such as cytokine receptors [3,4]. For the cytokine receptors, associated Janus kinases (JAKs) first phosphorylate the receptor itself and then the STATs [5]. Activated STATs form homo- or heterodimers and translocate into the nucleus where they bind to GAS (interferon$\gamma$ activated sequence) regulatory elements in the promoters of their target genes and then induce transcription [6]. They can be involved in the regulation of several important cellular processes such as cell growth, differentiation, or cell survival. Therefore, dysregulation of STATs involved in these pathways can lead to cellular transformation $[7,8]$. STATs that usually play a role in the promotion or suppression of malignant transformations are STAT1, -3 and, -5 . STAT1 exerts tumor suppressive functions such as induction of apoptosis or growth arrest, and also contributes to tumor rejection by the host $[9,10]$. On the other hand, both STAT5 proteins have been shown to be required for the development of myeloproliferative diseases [9]. 
STAT3 has also been implicated in malignant transformation and its suppression directs tumor cells to apoptosis $[11,12]$.

Chronic myeloid leukemia (CML) is a hematological cancer characterized by the accumulation of immature leukemic cells of myeloid origin in the bone marrow and bloodstream. The main driving force of this malignancy is the reciprocal translocation between the q arms of chromosomes 9 and 22, bringing the $B C R$ (breakpoint cluster region) and $A B L$ genes together $[13,14]$. The resultant chromosomal structure is referred to as the Philadelphia chromosome $(\mathrm{Ph})$, and the whole translocation process results in the expression of the $\mathrm{BCR} / \mathrm{ABL}$ fusion protein. Constitutive tyrosine kinase activity of BCR/ABL causes uncontrolled cell growth, and is also thought to be responsible for a variety of changes in normal cellular functions such as differentiation, adhesion, migration, and apoptotic response $[13,15]$. Thus, it has become an important target in the treatment of patients with Ph-positive CML. Imatinib was the first chemotherapeutic drug to be developed against $\mathrm{BCR} / \mathrm{ABL}$, and is still widely used in the first-line treatment of CML. Like other tyrosine kinases developed afterward, imatinib binds to the adenosine triphosphate (ATP)-binding domain of the fusion protein and prevents the subsequent phosphorylation of its target proteins, thereby blocking cell proliferation and inducing apoptosis [16]. Survival periods of patients with CML can be substantially prolonged by means of this drug, but in most cases acquired resistance can affect the clinical outcome of this therapy. Resistance is conferred by various mechanisms, one of which includes mutations in the drug-binding pocket of the BCR/ABL fusion protein $[17,18]$. Other mechanisms, such as fusion gene amplification (and therefore increased expression of the BCR/ABL protein) and overexpression of cellular efflux proteins, can diminish the bioavailability of the drug and hence contribute to resistance [19]. The involvement of tyrosine kinases other than BCR/ABL might also be responsible for providing resistance to leukemic cells [20].

Chemotherapeutic resistance is a major obstacle to maintaining effective cancer therapies. The administration of increased doses of chemotherapeutic drug in cases where resistant cancerous cells have developed is not always a good option, because of the serious side effects these drugs can have. Therefore, sensitizing these resistant cancerous cells back to these drugs might be a helpful way of gaining therapeutic success. Since STATs are implicated in the regulation of cell growth, manipulation of them might provide a good opportunity to achieve this goal. The aim of this study was therefore to investigate the differential expression pattern of STAT genes in imatinib-sensitive and -resistant K562 chronic myeloid leukemia cells, and especially to reveal the role of STAT5A after siRNA-mediated gene silencing followed by assessment of cell growth and apoptosis response.

\section{Methods}

Cell lines and culture conditions

Human chronic myeloid leukemia cells, K562, were obtained from the German Collection of Microorganism and Cell Cultures. Cells were grown in RPMI-1640 medium containing 10\% fetal bovine serum and $1 \%$ penicillin-streptomycin; and maintained at $37^{\circ} \mathrm{C}$ and in $5 \% \mathrm{CO}_{2}$. Imatinib-resistant K562 cells were developed by exposing them to increasing concentrations of imatinib for 2 years, starting with an initial concentration of $50 \mathrm{nM}$. Subpopulations of living cells were collected at each step. Resultant cells that had gained the ability to grow in the presence of $3 \mu \mathrm{M}$ imatinib are referred to as K562-IMA3 cells.

\section{Determination of differentially expressed STAT genes in imatinib-sensitive and-resistant cells by real-time $R T-P C R$}

mRNA expression levels of STAT3, STAT5A, and $S T A T 5 B$ genes were assessed by quantitative realtime reverse transcriptase-polymerase chain reaction (Q-PCR) initially, to reveal their differential expression patterns in imatinib-resistant K562-IMA3 cells. For this assessment, total RNA was isolated from both imatinib-sensitive and -resistant cells using the NucleoSpin ${ }^{\circledR}$ RNA II Kit (Macherey-Nagel, Easton, PA, USA) in accordance with the protocol provided by the manufacturer. Isolated RNAs were reverse transcribed into cDNAs, and Q-PCR was performed with gene-specific primers and probes using the LightCycler ${ }^{\circledR}$ Fast Start DNA Master HybProbes Kit and the LightCycler ${ }^{\circledR} 2.0$ Instrument (Roche Applied Science, Indianapolis, IN, USA). Glucose6-phosphate dehydrogenase $(G 6 P D H)$ was used as housekeeping gene in all PCR reactions, and its amplification product provided both a control for PCR performance and a reference for quantification of PCR products. Differential expression values were calculated from the standard curve obtained; for this purpose reference standards were used, starting from $5 \times 10^{6}$ mRNA copy numbers, decreasing to $5 \times 10^{2}$ mRNA copy numbers. The efficiency value calculated from this standard curve was 1.988 with an error of 0.01 , meaning that it could be used reliably in every PCR reaction. The relative expression level for 
each gene was calculated by dividing the mRNA copy number of the target gene by the G6PDH mRNA copy number of the reference gene.

\section{Gene silencing by siRNA transfection}

Since STAT5A was the gene whose expression levels changed most significantly in K562-IMA3 cells, we focused our interest on this. For its silencing, HiPerFect Transfection Reagent (Qiagen, Valencia, CA, USA) was used in combination with cholesterolconjugated anti-STAT5A siRNAs (Dharmacon, Chicago, IL, USA). The cholesterol conjugation resided at the $5^{\prime}$-end of the siRNA sense-strand, which specifically binds to STAT5A mRNAs. The reason why cholesterol-modified siRNAs were used is that they are more stable and, thus, more efficient in knocking down the target gene. Dulbecco's modified Eagle's medium (DMEM) was used for preparation of the transfection mixture. Eight hours after transfection cells were refreshed with new medium, and imatinib was added to the resistant cells to a final concentration of $3 \mu \mathrm{M}$ to maintain their previous culture conditions. In every experiment, also untreated resistant cells that served as a control were supplied with the specified concentration of imatinib. To reach the optimum silencing effect, cells were incubated totally for $72 \mathrm{~h}$ at $37^{\circ} \mathrm{C}$ and in $5 \% \mathrm{CO}_{2}$. Next, appropriate numbers of cells were collected for the XTT \{2,3-bis(2-methoxy-4nitro-5-sulfophenyl)-5-[(phenylamino) carbonyl]- $2 \mathrm{H}$ tetrazolium hydroxide $\}$ cell proliferation assay, and for Q-PCR analysis. Another round of transfection with new cells was also done for the caspase-3 activity assay. Again, $1 \times 10^{6}$ cells were allocated for the verification of silencing through Q-PCR analysis.

\section{Assessment of cellular proliferation by XTT}

K562 and K562-IMA3 cells were transfected with control and STAT5A siRNA and maintained in 96well plates with $100 \mu \mathrm{L}$ growth medium at a concentration of $2 \times 10^{4}$ cells/well in the absence or presence of increased doses of imatinib. After incubation at $37^{\circ} \mathrm{C}$ and in $5 \% \mathrm{CO}_{2}$ for $72 \mathrm{~h}$, XTT reagent (Cell Proliferation Kit II; Roche) was added into each well. Absorbance of a sample was measured by an enzyme-linked immunosorbent assay (ELISA) reader at $490 \mathrm{~nm}$ wavelength of light (Multiskan Spectrum; Thermo Electron Corporation, Vantaa, Finland) at the end of $4 \mathrm{~h}$ of incubation after adding XTT reagent. The obtained data were visualized by plotting cell proliferation curves, and $\mathrm{IC}_{50}$ (drug concentration that inhibits cell growth by 50\%) values were calculated for both sensitive and resistant cells.

\section{Measurement of caspase-3 enzyme activity}

Activity of the caspase- 3 enzyme was assessed at two different imatinib concentrations for each of the sensitive and resistant cells. Sensitive cells were treated with 100 and $200 \mathrm{nM}$ of imatinib, while concentrations of 5 and $10 \mu \mathrm{M}$ were selected for K562-IMA3 cells. Cells were incubated for $72 \mathrm{~h}$ at $37^{\circ} \mathrm{C}$ and in $5 \% \mathrm{CO}_{2}$ in the presence of the specified concentrations of imatinib. Assessment of caspase- 3 enzyme activity was done using the Caspase-3 Colorimetric Assay Kit (BioVision Research Products, Mountain View, CA, USA) according to the procedure provided by the manufacturer. In brief, cells were collected by centrifugation at $1000 \mathrm{~g}$ for $10 \mathrm{~min}$ and $100 \mu \mathrm{L}$ of cold lysis buffer was applied. Following the lysis step, cell debris were removed by centrifugation at $14000 \mathrm{~g}$ for $1 \mathrm{~min}$, and aliquots of the obtained protein mixture were taken for measuring the protein amount in extracts by bicinchoninic acid (BCA) assay. Protein mixtures were adjusted to equal concentrations according to results of the BCA assay, and the assessment of caspase- 3 enzyme activity was done by adding $5 \mu \mathrm{L}$ of the colorimetric caspase- 3 substrate, $20 \mu \mathrm{L}$ of assay buffer $(5 \times)$, and $50 \mu \mathrm{L}$ of distilled water to $25 \mu \mathrm{L}$ of protein samples in 96-well plates. To enable the enzymatic reaction, samples were maintained at $37^{\circ} \mathrm{C}$ for $2 \mathrm{~h}$ and sample absorbance was measured at 405 nm wavelength of light via an ELISA reader (Multiskan Spectrum; Thermo Electron Corporation).

\section{Results}

Differential expression patterns of STAT3, $-5 A$, and $-5 B$ in imatinib-sensitive and-resistant $\mathrm{K562}$ cells

Q-PCR analysis revealed that the expression level of STAT5A was most significantly changed among STATs examined in K562-IMA3 cells as compared to K562 cells (Figure 1). Its expression increased by $67 \%$, whereas the expression levels of STAT5B and STAT3 were increased $56 \%$ and $4 \%$, respectively. These results confirm observations from other studies, i.e. that activation of STAT3 is not important in the development of CML, and the present study now also shows that it does not play a role in the resistance mechanism developed against imatinib in CML. In the course of this study, we therefore mainly focused our interest on STAT5A, since its expression changed most in imatinib-resistant cells.

STAT5A knockdown in imatinib-sensitive and-resistant K562 cells

The efficacy of STAT5A knockdown by cholesterolconjugated anti-STAT5A siRNAs analyzed by 
Q-PCR was around $70-80 \%$ in imatinib-sensitive and -resistant K562 cells. In cells used for the XTT proliferation assay, STAT5A was suppressed by $71 \%$ and $69 \%$ for K562 and K562-IMA3, respectively (Figure 2); also, in cells used for caspase-3 activity assessment by its expression, STAT5A was suppressed by $80 \%$ and $71 \%$, respectively, compared to control groups.

\section{$X T T$ cell proliferation assay of non-transfected and siRNA-transfected cells}

XTT cell proliferation assays were performed using different concentrations of imatinib for K562 and K562-IMA3 cells. This resulted in cell proliferation curves that enabled the calculation of $\mathrm{IC}_{50}$ values. For non-transfected K562 and K562-IMA3 cells, imatinib $\mathrm{IC}_{50}$ values were calculated as $280 \mathrm{nM}$ and $14761 \mathrm{nM}$, respectively (Figures 3 and 4); on the other hand, $\mathrm{IC}_{50}$ values for cholesterol-conjugated anti-STAT5A siRNA-transfected K562 and K562-
IMA3 cells were $248 \mathrm{nM}$ and $3285 \mathrm{nM}$, respectively (Figures 3 and 4). Taken together, these results indicated that silencing of STAT5A had sensitized both sensitive and resistant K562 cells to imatinib. siRNA-transfected resistant cells became almost 4.5 times more sensitive to imatinib than their nontransfected counterparts, while transfected sensitive cells showed approximately 1.12-fold increased sensitivity.

\section{Assessment of caspase-3 enzyme activity}

Activation of caspase- 3 is particularly important for the execution of apoptosis. For this reason, we assessed the activation of this enzyme to investigate the apoptosis induction of cells upon treatment with imatinib, before and after anti-STAT5A siRNA transfection. Enhanced apoptosis induction was concurrent with the suppression of STAT5A expression in both imatinib-sensitive and -resistant K562 cells. For K562-IMA3 cells, there were

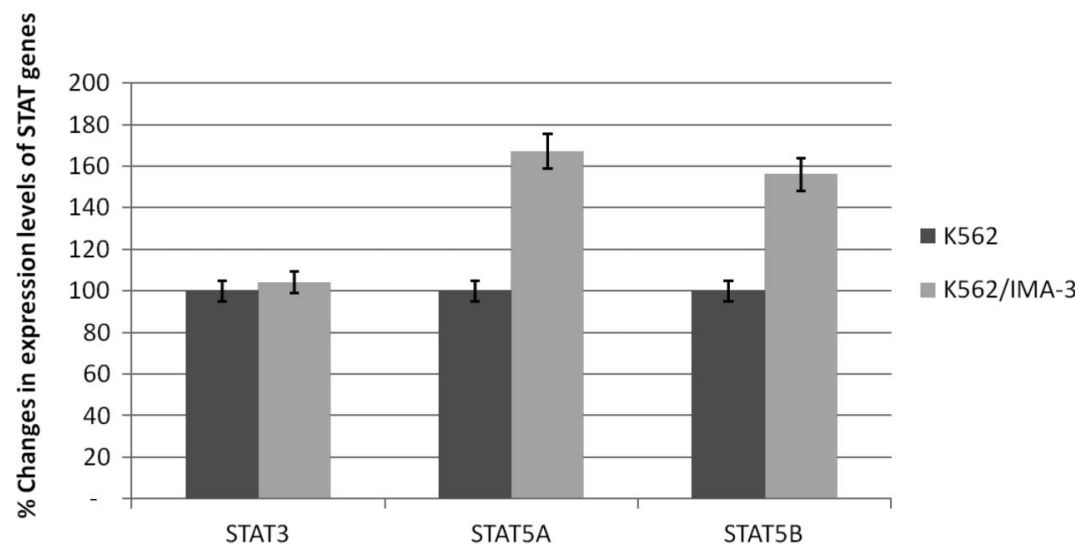

Figure 1. Differential expression of STAT genes in K562 and K562-IMA3 cells. Expression of each of the genes was normalized to $100 \%$ for K562 cells and then K562-IMA3 data were plotted relative to that normalization. Error bars demonstrate standard deviations and $p<0.05$ was considered as statistically significant.

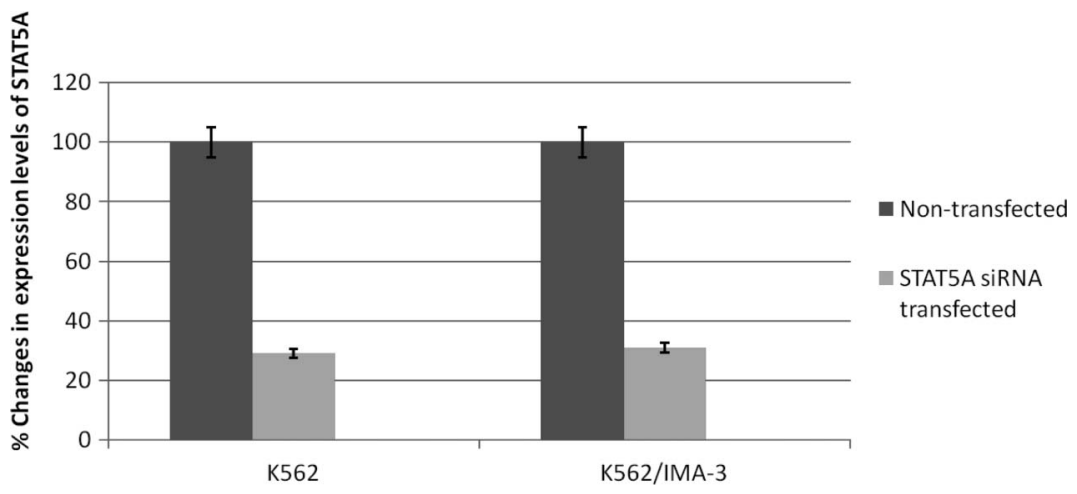

Figure 2. Expression levels of STAT5A in non-transfected and STAT5A siRNA-transfected cells before proceeding with XTT proliferation assay and caspase-3 enzyme activity assessment. Non-transfected K562 and K562-IMA3 data were normalized to 100\% separately and expression levels after siRNA transfection are depicted relative to these. Error bars indicate standard deviations of measurements and $p<0.05$ was considered as significant. 
dose-dependent increases in caspase- 3 enzyme activity in non-transfected and siRNA-transfected samples (Figure 5). Non-transfected K562-IMA3 cells

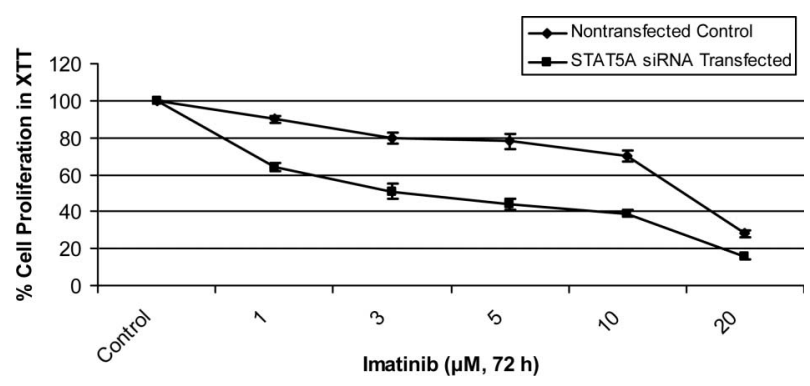

Figure 3. Effect of imatinib on the growth of STAT5A siRNAtransfected and non-transfected K562-IMA3 cells. Error bars indicate standard deviations in the experiment, and when not seen, they are smaller than the proliferation curve itself. Similar to the previous analyses, $p<0.05$ was defined as statistical significance.

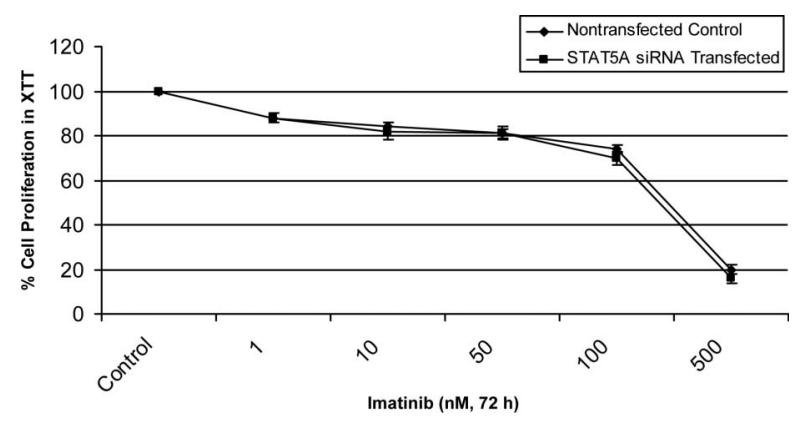

Figure 4. Effect of imatinib on the growth of STAT5A siRNAtransfected and non-transfected K562 cells. Error bars indicate standard deviations in the experiment, and if they are not apparent, the thickness of the bar is less than the proliferation curve itself. As in the previous analyses, $p<0.05$ was defined as statistical significance. showed 1.85- and 3.46-fold increases in caspase-3 enzyme activation in the presence of 5 and $10 \mu \mathrm{M}$ imatinib, while siRNA-transfected counterparts showed 11.21- and 20.17-fold increases. These results are in accordance with the XTT data obtained for K56-IMA3 cells as noted in the previous section. Non-transfected sensitive K562 cells also showed dose-dependent increases in caspase- 3 enzyme activities, which were 2.95- and 3.38-fold in the presence of 100 and $200 \mathrm{nM}$ imatinib. However, siRNAtransfected sensitive K562 cells demonstrated an unusual pattern: they showed a decrease in caspase- 3 enzyme activity by 0.83 -fold in the presence of 100 $\mathrm{nM}$ imatinib, but a 20.18-fold increase in the presence of $200 \mathrm{nM}$ imatinib (Figure 6).

\section{Discussion}

In this study, the effects of STAT5A silencing on chemotherapeutic cell death and resistance were investigated. Suppression of STAT5A expression was achieved by transfection of cholesterol-conjugated anti-STAT5A siRNAs into human chronic myeloid leukemia cells that were either sensitive or resistant to imatinib, a $\mathrm{BCR} / \mathrm{ABL}$ tyrosine kinase inhibitor. Our experimental results have shown that diminution of STAT5A expression evokes increased induction of apoptosis and enhanced sensitivity to imatinib, therefore making it important for leukemic cell survival.

At the initial stage of our study we found that the most significant changes in gene expression happened for STAT5A in imatinib-resistant K562 chronic myeloid leukemia cells, possibly indicating its importance in this process rather than of STAT3 (since the amount of $S T A T 3$ expression was much less than of $S T A T 5 A$ and $S T A T 5 B$ ). Leukemic cells

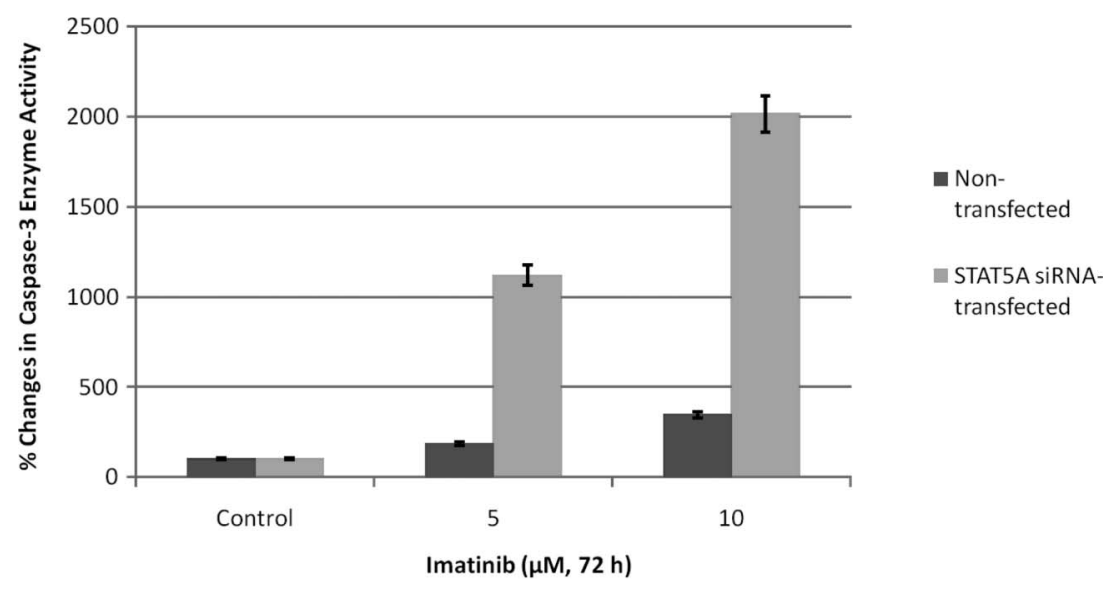

Figure 5. Percent changes in caspase-3 enzyme activity in non-transfected and STAT5A siRNA-transfected K562-IMA3 cells. Data were normalized to $100 \%$ for non-transfected and siRNA-transfected results separately for control samples, and data points are depicted relative to those normalizations. Error bars show standard deviations in the experiment. Values of $p<0.05$ were considered as statistically significant. 


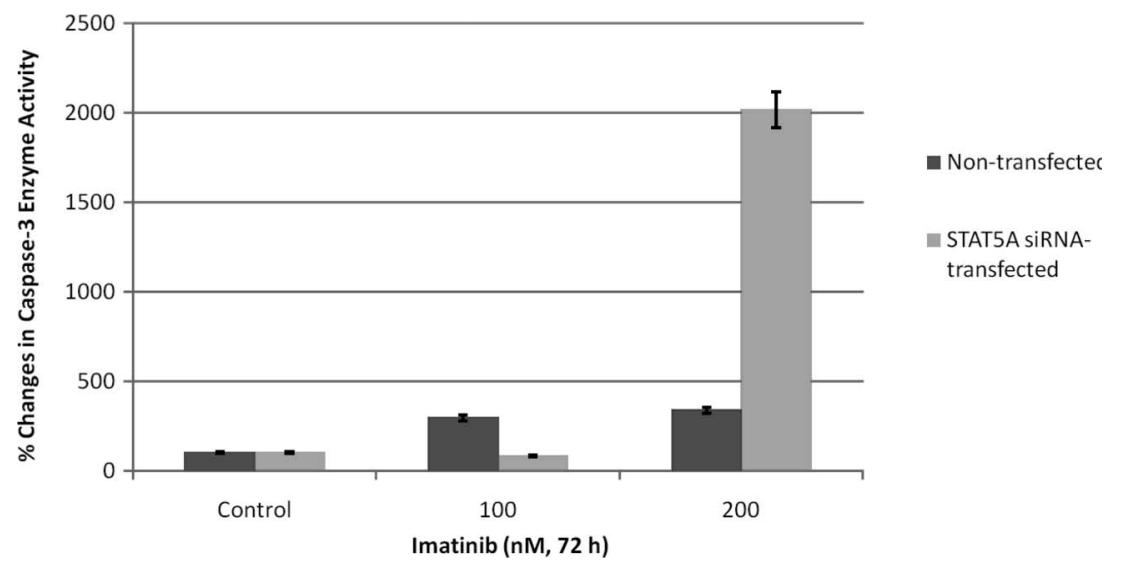

Figure 6. Percent changes in caspase-3 enzyme activity in non-transfected and STAT5A siRNA-transfected K562 cells. Data were normalized to $100 \%$ for non-transfected and siRNA-transfected results separately for control samples, and data points are depicted relative to those normalizations. Error bars show standard deviations in the experiment. Values of $p<0.05$ were considered as statistically significant.

usually develop multiple features to cope with the stress of chemotherapy, one of which is amplification of the fusion protein. Our previous studies have shown that an elevated level of BCR/ABL is an important factor in conferring chemotherapeutic resistance to CML cells [17]. There are studies indicating that $\mathrm{BCR} / \mathrm{ABL}$ induces activation of STAT5 [21,22], and from this perspective, our findings are in accordance with the previous literature. This situation might be useful in explaining the difference in chemotherapeutic sensitization of imatinib-sensitive and -resistant cells upon siRNA transfection. Resistant cells became approximately 4.5 times more sensitive, while sensitive cells showed around 1.12-fold increased sensitivity. If the increased activation of STAT5A is related to the achievement of resistance, then silencing of STAT5A would have more profound effects on K562-IMA3 cells than on imatinib-sensitive K562 cells, whose STAT5A expression remained diminutive compared to the high expression in resistant cells. These significant results were obtained when the silencing efficiency of STAT5A was around $70 \%$. By further experimentation with increased silencing efficiencies, these differences might become even higher between the imatinib-sensitive and -resistant CML groups.

When caspase- 3 enzyme activity test results were examined, generally an imatinib dose-dependent increase was observed. However, siRNA-transfected K562 cells showed a relatively different pattern, with a slight decrease at $100 \mathrm{nM}$ of imatinib and around 20fold increase at the next drug concentration, which was $200 \mathrm{nM}$. This pattern might be attributed to a delayed response to the drug, but does not seem particularly important since there was a more than 20-fold increase at the next concentration. When compared to the 200 $\mathrm{nM}$ data, the magnitude of decrease also seemed not so significant. However, to further investigate whether such a pattern of decrease occurs before that particular data point, additonal caspase- 3 enzyme activity experiments might be performed, involving smaller drug concentrations. Imatinib, as part of its mechanism of action, blocks activity of the BCR/ABL fusion protein by specifically binding to its ATP-binding domain, leading to apoptotic cell death by this means. Increased caspase- 3 enzyme activity showed that cells were still dying through apoptosis in the presence of imatinib upon transfection with cholesterol-conjugated anti-STAT5A siRNAs.

These results may have clinical importance, too, since drug resistance is one of the major problems in cancer therapy. Through our experiments, we have shown that resistant CML cells could be sensitized to obtain more effective chemotherapeutic responses. One may speculate that the $\mathrm{IC}_{50}$ value of siRNAtransfected resistant cells is still high (especially when compared to the $\mathrm{IC}_{50}$ value of sensitive cells), and therefore this approach is not feasible enough for a clinical impact. However, the following explanations might provide answers to these concerns. Our first explanation involves the efficiencies of transfection and gene silencing. These results were obtained when STAT5A expression was silenced around 70$80 \%$. Silencing with increased efficiency or complete removal of the gene by homologous recombination might provide better responses to imatinib in resistant cells. The second explanation could be that other STAT family members might physiologically make up the loss of STAT5A activity. STAT3 and STAT5B are good candidates for such a mechanism, since they are frequently found to be overexpressed in various cancers $[23,24]$. Because of the high gene homology it shares with STAT5A, STAT5B may be involved with it in redundant functions [25], for example reducing the inhibition of cell growth, similar to STAT5A. It might be 
interesting to investigate cell growth under the application of chemotherapy when both STAT5 genes are simultaneously suppressed. Also, experiments investigating the silencing effects of STAT genes on cell growth might be conducted with other chemotherapeutic agents to obtain detailed information about the relationship between these genes and multi-drug resistance. Nevertheless, observing these significant responses to imatinib after transfection with single gene-specific siRNAs might provide new opportunities for dealing with the frequent occurrence of resistance to chemotherapeutic agents in leukemia.

\section{Acknowledgement}

We thank the staff of the Biotechnology and Bioengineering Center of the Izmir Institute of Technology for their help and technical support.

Declaration of interest: This work was supported by The Scientific and Technological Research Council of Turkey and by the Turkish Society of Hematology (TUBITAK 105S459 and THD 20002/08, respectively, to $\mathrm{BK}$ ).

\section{References}

1. Darnell JE Jr, Kerr IM, Stark GR. Jak-STAT pathways and transcriptional activation in response to IFNs and other extracellular signaling proteins. Science 1994;264: $1415-1421$.

2. Schindler C, Darnell JE Jr. Transcriptional responses to polypeptide ligands: the JAK-STAT pathway. Annu Rev Biochem 1995;64:621-651.

3. Leaman DW, Leung S, Li X, Stark GR. Regulation of STATdependent pathways by growth factors and cytokines. FASEB J 1996;10:1578-1588.

4. Ihle JN, Witthuhn BA, Quelle FW, Yamamoto K, Silvennoinen O. Signaling through the hematopoietic cytokine receptors. Annu Rev Immunol 1995;13:369-398.

5. Darnell JE Jr. STATs and gene regulation. Science 1997;277:1630-1635.

6. Ramana CV, Grammatikakis N, Chernov M, et al. Regulation of c-myc expression by IFN-gamma through Stat1-dependent and -independent pathways. EMBO J 2000;19:263-272.

7. Shang Y, Kakinuma S, Amasaki Y, et al. Aberrant activation of interleukin-9 receptor and downstream $S$ tat $3 / 5$ in primary $\mathrm{T}$ cell lymphomas in vivo in susceptible $\mathrm{B} 6$ and resistant $\mathrm{C} 3 \mathrm{H}$ mice. In Vivo 2008;22:713-720.

8. Momose S, Tamaru J, Kishi H, et al. Hyperactivated STAT3 in ALK-positive diffuse large B-cell lymphoma with clathrinALK fusion. Hum Pathol 2009;40:75-82.
9. Levy DE, Gilliland DG. Divergent roles of STAT1 and STAT5 in malignancy as revealed by gene disruptions in mice. Oncogene 2000;19:2505-2510.

10. Bromberg JF, Horvath CM, Wen Z, Schreiber RD, Darnell JE Jr. Transcriptionally active Stat 1 is required for the antiproliferative effects of both interferon alpha and interferon gamma. Proc Natl Acad Sci USA 1996;93:7673-7678.

11. Bromberg J. Stat proteins and oncogenesis. J Clin Invest 2002;109:1139-1142.

12. Catlett-Falcone R, Landowski TH, Oshiro MM, et al. Constitutive activation of Stat3 signaling confers resistance to apoptosis in human U266 myeloma cells. Immunity 1999;10:105-115.

13. Deininger MW, Goldman JM, Melo JV. The molecular biology of chronic myeloid leukemia. Blood 2000;96:33433356.

14. Koca E, Haznedaroglu IC. Imatinib mesylate and the management of chronic myeloid leukemia (CML). Turk J Hematol 2005;22:161-172.

15. Peled A, Hardan I, Trakhtenbrot L, et al. Immature leukemic CD34+CXCR4+ cells from CML patients have lower integrin-dependent migration and adhesion in response to the chemokine SDF-1. Stem Cells 2002;20:259-266.

16. Schindler T, Bornmann W, Pellicena P, et al. Structural mechanism for STI-571 inhibition of abelson tyrosine kinase. Science 2000;289:1938-1942.

17. Baran Y, Ural AU, Gunduz U. Mechanisms of cellular resistance to imatinib in human chronic myeloid leukemia cells. Hematology 2007;12:497-503.

18. Frame D. New strategies in controlling drug resistance. J Manag Care Pharm 2007;13:13-17.

19. Illmer T, Schaich M, Platzbecker U, et al. P-glycoproteinmediated drug efflux is a resistance mechanism of chronic myelogenous leukemia cells to treatment with imatinib mesylate. Leukemia 2004;18:401-408.

20. Dai Y, Rahmani M, Corey SJ, Dent P, Grant S. A Bcr/Ablindependent, Lyn-dependent form of imatinib mesylate (STI571) resistance is associated with altered expression of Bcl-2. J Biol Chem 2004;279:34227-34239.

21. Carlesso N, Frank DA, Griffin JD. Tyrosyl phosphorylation and DNA binding activity of signal transducers and activators of transcription (STAT) proteins in hematopoietic cell lines transformed by Bcr/Abl. J Exp Med 1996;183:811-820.

22. Chai SK, Nichols GL, Rothman P. Constitutive activation of JAKs and STATs in BCR-Abl-expressing cell lines and peripheral blood cells derived from leukemic patients. J Immunol 1997;159:4720-4728.

23. Weber-Nordt RM, Egen C, Wehinger J, et al. Constitutive activation of STAT proteins in primary lymphoid and myeloid leukemia cells and in Epstein-Barr virus (EBV)-related lymphoma cell lines. Blood 1996;88:809-816.

24. Garcia R, Yu CL, Hudnall A, et al. Constitutive activation of Stat 3 in fibroblasts transformed by diverse oncoproteins and in breast carcinoma cells. Cell Growth Differ 1997;8:12671276.

25. Teglund S, McKay C, Schuetz E, et al. Stat5a and Stat5b proteins have essential and nonessential, or redundant, roles in cytokine responses. Cell 1998;93:841-850. 